\title{
Preliminary analysis of pollution characteristics and sources in a typical city in Jiangsu Province
}

\author{
Zhicheng Zhou ${ }^{1, a}$, Yong Sun ${ }^{2, b}$,Di Cheng ${ }^{3, c}$, Xiaoyu Zhou ${ }^{4, d}$ \\ ${ }^{1}$ Electric power research institute of Jiangsu electric power company, Nanjing 211103,China; \\ ${ }^{2}$ Nanjing University of Information Science and Technology, Nanjing 210044,China; \\ ${ }^{3}$ Nanjing University of Information Science and Technology, Nanjing 210044,China; \\ ${ }^{4}$ Nanjing University of Information Science and Technology, Nanjing 210044,China; \\ asimencola@sohu.com, b925726321@qq.com, c281559315@qq.com,d245144672@qq.com
}

Keywords: Jiangsu Province; typical city; pollution characteristics

Abstract The distribution of pollutants in 13 cities in Jiangsu Province in the winter of 2014 was analyzed. It was found that there were more haze days in Nanjing and Xuzhou and the concentration of $\mathrm{PM}_{10}$ and $\mathrm{PM}_{2.5}$ exceeded the national standard $38.9 \%$ and $42.2 \%$ respectively in the Nanjing, $51.1 \%$ and $55.6 \%$ respectively in Xuzhou. Nanjing and Xuzhou are affected by both local and external sources. The southward airflow is easy to accumulate pollution in Nanjing and Xuzhou, while the northeastern airflow and precipitation are favorable for the removal of pollutants. Large, and Nanjing area by local and external sources of common influence.

\section{Introduction}

In recent years, haze has led to an increase of low visibility events, The increase in haze phenomenon is related to the high solubility of aerosol particles in China ${ }^{[1]}$.

The important precursor contaminants of forming secondary aerosol particles is primary particles and the chemical reaction of gas pollutants in atmosphere, One of the most important is the photochemical reaction ${ }^{[2]}$.Through this process is mainly produced by fine particles,.It is difficult to remove by gravity sedimentation and turbulent transport process , the fine particles in air is easy to accumulate in a more stable atmosphere,resulting in decreased visibility, thus forming haze pollution ${ }^{[3]}$. As the haze visibility is very low, sunshine gradually reduced or no sunshine, people's health, greenhouse production and transportation have caused a very bad impact ${ }^{[4]}$, Therefore, it is necessary to study the characteristics of pollutants in haze pollution.

In this paper, the relationship between the change of atmospheric pollutants and the meteorological factors in Nanjing and Xuzhou which haze is not alleviated and pollution is more serious are analyzed by using meteorological regular observation data and air pollutant concentration data for the winter of 2014 in Jiangsu Province and the relationship between the change of atmospheric pollutants and the meteorological factors is analyzed. Wind field analysis of the source of pollutants, in-depth understanding of the pollution situation in Nanjing and Xuzhou City, for the two cities to develop practical pollution prevention measures to provide effective support. Combined with the wind field analysis of the source of pollutants, understand the pollution situation in Nanjing and Xuzhou City deeply, Provide effective support for the two cities to develop practical pollution prevention measures.

\section{Data}

\section{Site data}

In this paper, 72 basic weather stations and 63 national control environmental monitoring stations in Jiangsu Province were selected. Observational data is from October 2014 to April 2015, of which the conventional meteorological observation data time resolution of $10 \mathrm{~min}$, air pollutant concentration data time resolution of $1 \mathrm{~h}$. 


\section{Distribution characteristics of haze in Jiangsu Province}

Using haze day discrimination method analys of haze in Jiangsu Province.In the past, In Meteorological regulations ,the main basis discrimination of haze day is based on relative humidity and visibility, when the visibility less than $10 \mathrm{~km}$ under the premise, Relative humidity will be the haze of the criteria. National Meteorological Administration promulgated the "People's Republic of Meteorological Industry Standard" [5] in 2010. In this standard, the daily average visibility is less than $10 \mathrm{~km}$, Daily relative humidity less than $80 \%$ or daily relative humidity between $80 \%-95 \%, \mathrm{PM}_{2.5}$ concentration greater than $75 \mu \mathrm{g} \cdot \mathrm{m}^{-3}$ will be judged as a haze day

Atmospheric visibility is usually defined as the maximum distance that can be seen and identified in the surrounding environment,it's an important indicator of the haze day ${ }^{[6-7]}$.

Table 1 The statistics of haze days (the total period of 182 in winter 2014-2015)

\begin{tabular}{llllllllllllll}
\hline City & NJ & ZJ & CJ & WX & SZ & YZ & TZ & NT & HA & YC & XZ & SQ & LYG \\
& & & & & & & & & & & & & \\
\hline Haze day & 132 & 122 & 126 & 131 & 124 & 128 & 123 & 108 & 129 & 125 & 142 & 133 & 119 \\
& & & & & & & & & & & & & \\
& & & & & & & & & & & & & \\
\hline Percent & $73 \%$ & $67 \%$ & $69 \%$ & $72 \%$ & $68 \%$ & $70 \%$ & $68 \%$ & $59 \%$ & $71 \%$ & $69 \%$ & $78 \%$ & $73 \%$ & $65 \%$
\end{tabular}

In table 1, the coastal areas are less foggy days, perhaps because coastal areas will have an injection from the sea clean air, so the air in the coastal areas is cleaner than in other areas. In the period from October 2014 to April 2015, the maximum number of haze days in the eight cities in southern Jiangsu was 132 days, and the lowest in Nantong in coastal areas was 108 days. In the same time, the most smog days in five cities in northern Jiangsu were 142 days in Xuzhou, the second is 133 days in Suqian, and the rest cities is about 120 days, and the lowest in Lianyungang coastal area was 119 days. According to the above analysis, there are more smog days in Nanjing and Xuzhou city, so these two cities are selected as the typical cities with more serious pollution.

\section{The distribution of pollution characteristics and the influence of meteorological factors in Nanjing and Xuzhou}

\section{Monthly Variation of Air Pollutant Concentration in Nanjing and Xuzhou}

Fig. 1 is a monthly change cabinet diagram of the daily mean of pollutant concentration in Nanjing, including monthly mean, maximum value, minimum value, and $75 \%, 25 \%$ quantile. The results showed that $\mathrm{O}_{3}$ concentration decreased gradually from October to January, and the concentrations of co, $\mathrm{SO}_{2}, \mathrm{PM}_{10}$ and $\mathrm{PM}_{2.5}$ were gradually increased, and the daily mean concentration was most discrete in January, indicating that there were higher and lower values in January, and the seasonal variation of $\mathrm{NO}_{2}$ was relatively small. From February, the concentrations of co, $\mathrm{PM}_{10}$ and $\mathrm{PM}_{2.5}$ decreased gradually, especially the median daily mean concentration of $\mathrm{PM}_{10}$ was larger than that in January and February. the median daily mean concentration of $\mathrm{PM}_{2.5}$ was not significantly higher than that in January and February, indicating that the main pollutants were coarse particulate matter in January, which contributed to the conversion of gas to particulate matter after February with the increase of precipitation. Since February wind speed is relatively large and easterly wind blowing, the east wind can bring the clean air mass on the sea to land, diluting the concentration of the pollutants, leading to the lower concentration of $\mathrm{O}_{3}$, and the chemical loss reduced, so as to start in February, $\mathrm{O}_{3}$ concentration

gradually 
increased.
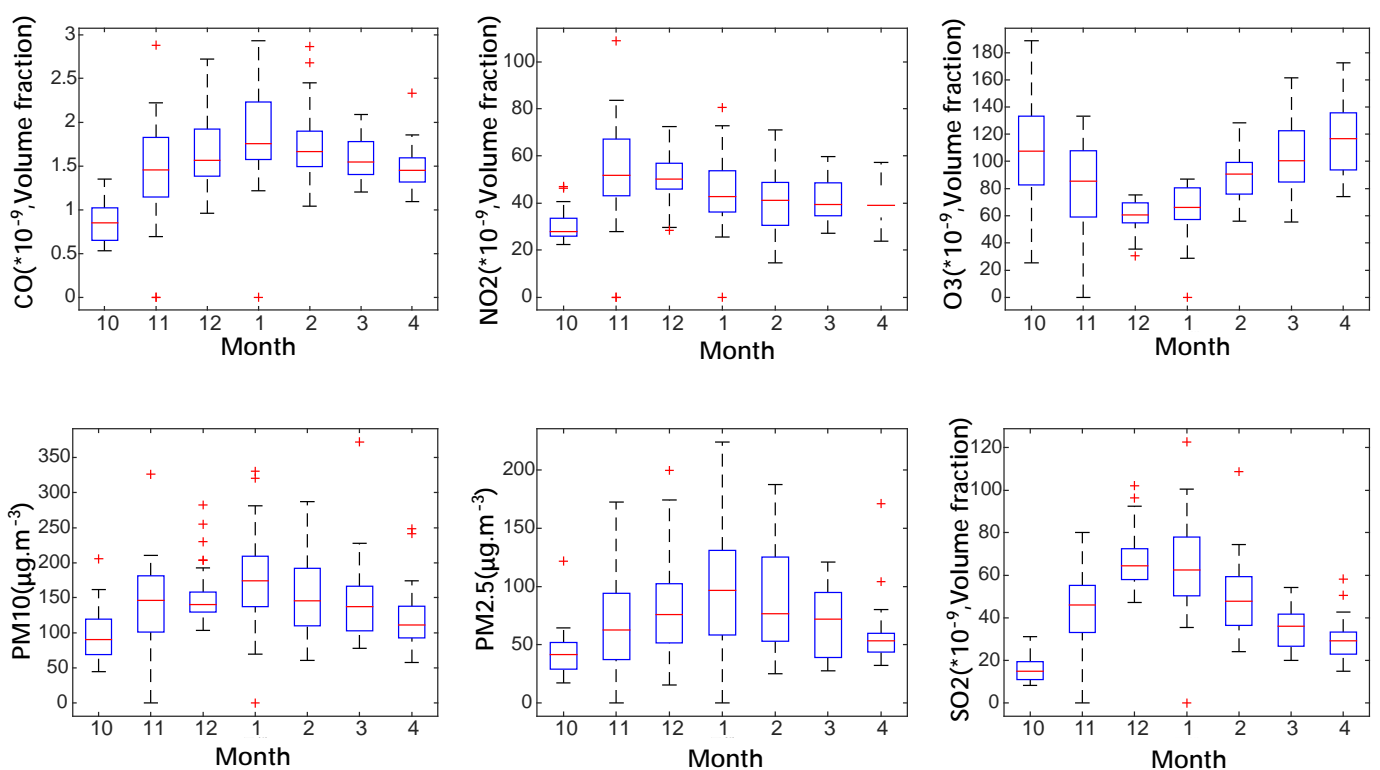

Fig. 1 Diurnal variation of pollutant concentration in Nanjing city

Figure 2 shows the monthly variation of the daily mean of pollutant concentrations in Xuzhou, the box diagram includes monthly mean, maximum, minimum, $75 \%$ and $25 \%$ quantiles. The results are similar to those in the Nanjing, but the data are more concentrated, indicating that the daily mean of pollutants per month is not much different. The concentration of $\mathrm{PM}_{10}$ in Xuzhou is greater than Nanjing, $\mathrm{PM}_{10}$ pollution is more serious, and the concentration of $\mathrm{NO}_{2}$ is different from Nanjing, the highest concentration appeared in December, January is a relatively serious month of pollution in Xuzhou, the monthly mean values of $\mathrm{CO}, \mathrm{NO}_{2}, \mathrm{PM}_{10}, \mathrm{PM}_{2.5}$ and $\mathrm{SO}_{2}$ were $1.8\left(* 10^{-9}\right), 41\left(* 10^{-9}\right)$, $176 \mu \mathrm{g} / \mathrm{m}^{-3}, 98.7 \mu \mathrm{g} / \mathrm{m}^{-3}$ and $62\left(* 10^{-9}\right)$, respectively.
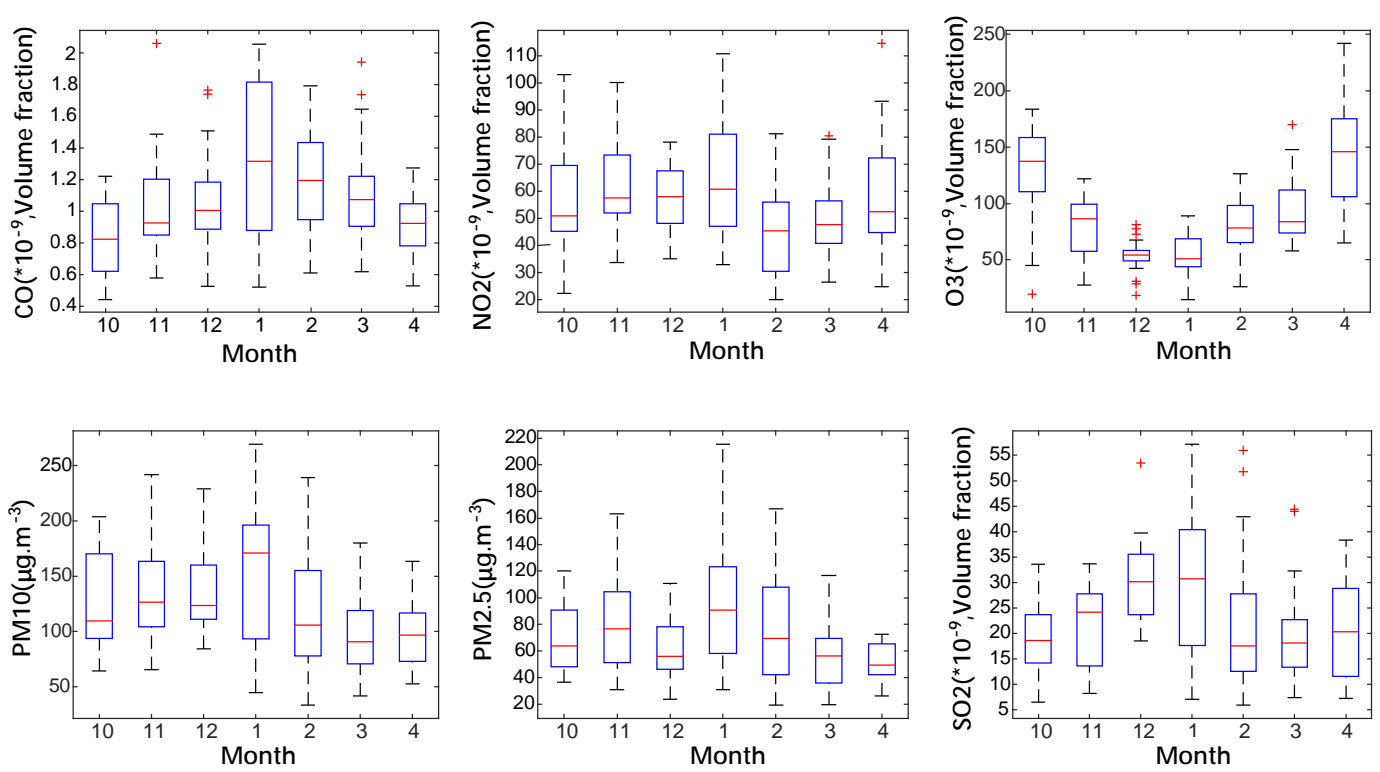

Fig. 2 Diurnal variation of pollutant concentration in Xuzhou city 


\section{Time Series Changes of Atmospheric Pollutants and Meteorological Factors in Nanjing and Xuzhou City}

Figure 3 and Figure 4, respectively, Nanjing and Xuzhou City, the concentration of pollutants and meteorological elements of daily changes. It can be seen the maximum precipitation in late November and mid-March, and there is little precipitation at other times, and there is a significant decrease in the concentration of pollutants in precipitation, indicating the clear effectiveness of wet deposition on pollutants. At the same time, in the relatively high temperature months, such as October, April. $\mathrm{O}_{3}$ content is relatively high, mainly because $\mathrm{O}_{3}$ is by the NOx and VOCs in the ultraviolet light under the action of photochemical reactions associated. The temperature is low, radiation is small and is not conducive to $\mathrm{O}_{3}$ generation in January and February. While the $\mathrm{SO}_{2}$ and $\mathrm{NO}_{2}$ contents were higher in January and February, and the temperature was lower and the precipitation was less correlated. At the same time, when the wind speed is large, the pollutant concentration is low, which indicates that the static wind condition is an important reason for the high concentration of pollutants. In summary, small winds and precipitation are important weather conditions that lead to high concentrations of pollutants.
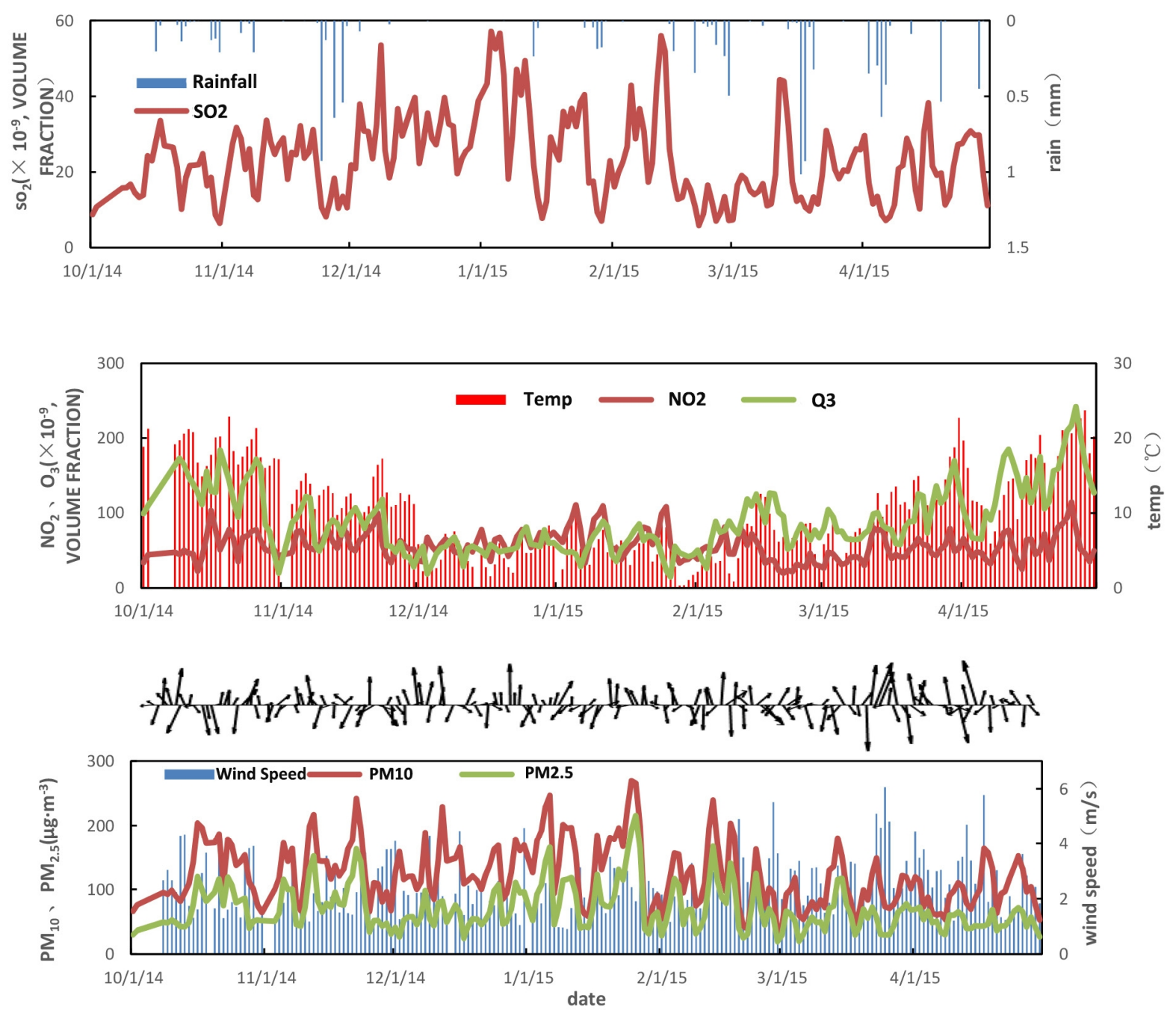

Fig.3 Diurnal variation of pollutant concentration and meteorological factors in Nanjing 

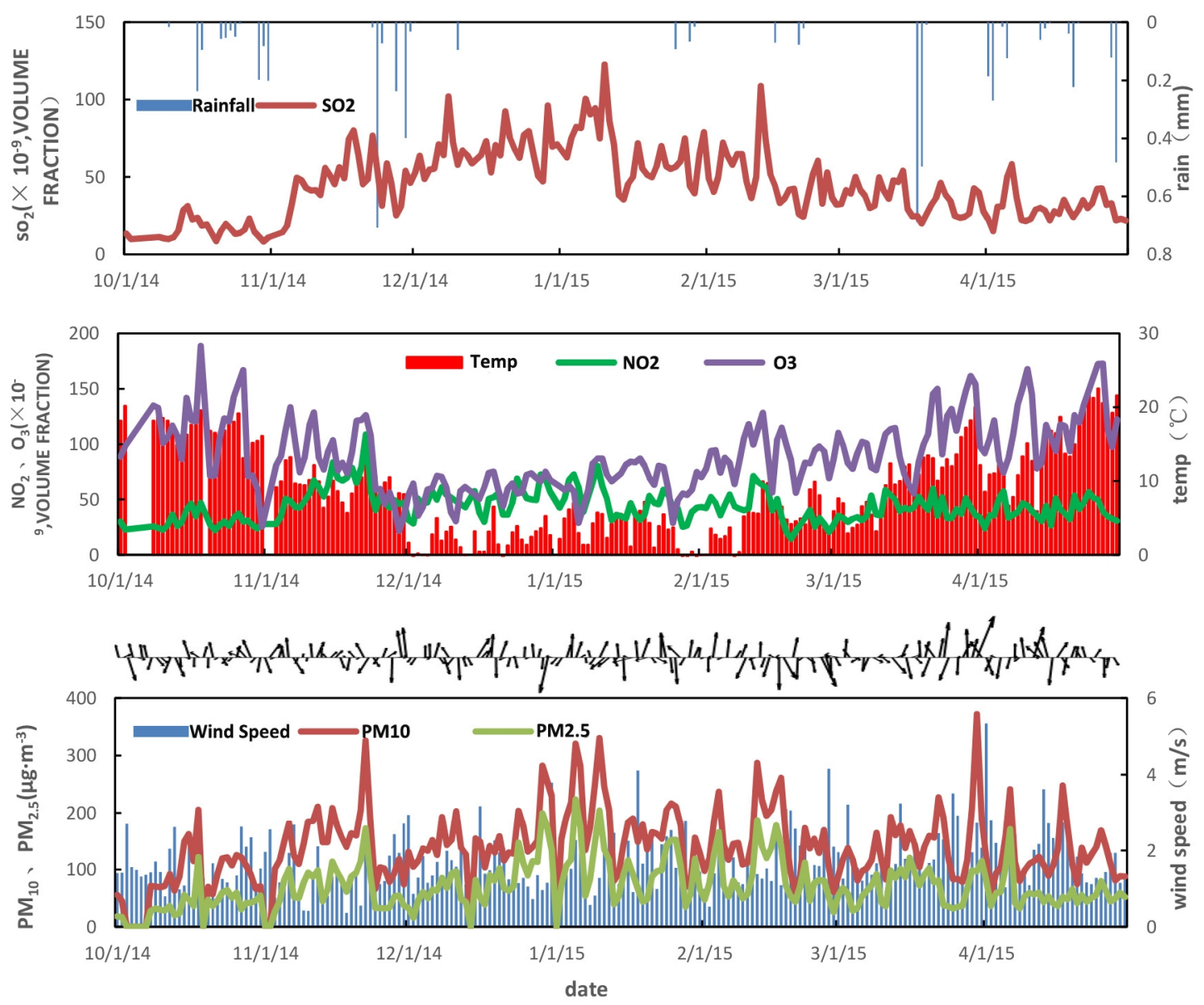

Fig.4 Diurnal variation of pollutant concentration and meteorological factors in Xuzhou City

\section{Conclusions}

In this paper, the characteristics of pollution characteristics and the influence of meteorological factors on the two cities with more haze in Jiangsu Province, Nanjing and Xuzhou are studied, and the following conclusions are drawn:

(1) haze in the coastal areas is less than inland, taking into account the coastal areas tend to have from the sea clean air injection, so that coastal areas than the other areas of air clean. In the period from October 2014 to April 2015, the number of days of haze in the eight cities in southern Jiangsu was 132 days, and the haze of Nantong in coastal areas was at least 108 days. At the same time, In the city, Xuzhou haze day up to 142 days, followed by Suqian, its haze day is 133 days, the rest of the city are about 120 days, the coastal area of Lianyungang haze day at least 119 days.

(2) Nanjing and Xuzhou City are affected by local and external pollutants, the south side of the air can easily lead to the accumulation of pollution in Nanjing and Xuzhou City, while the northeastern airflow and precipitation is conducive to the removal of pollutants, Xuzhou area by the local Pollution is greater, and Nanjing area by local and external sources of common influence. 


\section{References}

[1] Zhang Xiaotong, Sun Junying, Wang Yajiang, Li Weijun, Zhang Qiang, Wang Weigang, Quan Jiannong, Cao Guoliang, Wang Jizhi, Yang Yuanqin, Zhang Yangmei. Chinese Fog - haze Cause and Its Governance $[\mathrm{J}]$ Journal of Science and Technology, Nanjing University of Aeronautics and Astronautics. 2013, 13: 1178-1187.

[2] Zhu Bin, An Junling, Wang Zifa, Li Yi. Discussion on the diurnal variation of photochemical ozone and its precursors [J]. Journal of Nanjing Institute of Meteorology, 2006,06: 744-749.

[3] Mao Minjuan, Liu Houtong, Xu Honghui, Pu Jingjiao. Study on the Key Cause of Haze in the Application of Multivariate Observation Data Fusion [J]. Chinese Journal of Environmental Science, 2013,03: 806-813.

[4] Zheng Qiuping, Liu Hongnian, Zhang Lijuan, Zhu Yan, Zhu Lian-fang, Jiang Wei-mei. Evaluation of haze in Suzhou [J]. Meteorological Science, 2013,01: 83-88.

[5] Sun Rui. Improvement of haze meteorological factors in Beijing area and improvement of discriminant criteria [D]. Nanjing University of Information Science and Technology, 2015.

[6]World Meteorological Organization, WMO-No. 266, Compendium of Lecture Notes for Training Class IV Meteorological Personnel Volume I I-Meteorology [S ] .

[7] Meteorological Office, Handbook of Aviation Meteorology [S] . 